\title{
Intestinal microbiota mediates the beneficial effects of $n-3$ polyunsaturated fatty acids during dietary obesity
}

\author{
Jérôme Bellenger ${ }^{1,2,3,{ }^{*}}$, Sandrine Bellenger ${ }^{1,2,3}$, Amina Bourragat ${ }^{1,2,3}$, Quentin Escoula ${ }^{1,2,3,4}$, \\ Pierre Weill ${ }^{4}$ and Michel Narce ${ }^{1,2,3}$ \\ ${ }^{1}$ Université de Bourgogne Franche-Comté, UFR Sciences de la Vie, de la Terre et de l'Environnement, Lipides Nutrition Cancer UMR \\ UMR1231, 6 Boulevard Gabriel, 21000 Dijon, France \\ 2 INSERM, Lipides Nutrition Cancer UMR1231, 21000 Dijon, France \\ ${ }^{3}$ LipSTIC LabEx, Fondation de Coopération Scientifique Bourgogne-Franche Comté, 21000 Dijon, France \\ ${ }^{4}$ Valorex, La Messayais, 35210 Combourtillé, France
}

Received 11 September 2020 - Accepted 22 January 2021

\begin{abstract}
Obesity, now considered as a real worldwide epidemic affecting more than 650 million people, is complex and mainly associated with excessive energy intake and changes in eating habits favoring the consumption of diets rich in saturated fat and sugar. This multifactorial pathology is linked to chronic low grade systemic inflammation. Indeed, a high fat diet (HFD) leads to intestinal microbiota dysbiosis increasing gut permeability (partly attributed to a downregulation of genes encoding tight junction proteins) leading to an increase in bacterial lipopolysaccharides (LPS) levels so-called metabolic endotoxemia. Studies have shown that $\mathrm{n}-3$ polyunsaturated fatty acids (PUFAs) are involved in the prevention of obesity and insulin resistance partly through synthesis of lipid mediators. While studies suggest that $n-3$ PUFAs are able to modulate the gut microbiota, others show no effect of n-3 treatments on intestinal homeostasis. In the present work, we showed that when fed a hypercaloric and obsogenic diet, compared with wild-type (WT) mice, fat-1 mice (with constitutive production of n-3 PUFAs) resist to dietary obesity and associated metabolic disorders, maintain an effective gut barrier function and exhibit greater phylogenic diversity. Moreover, fecal microbiota transplantation from fat-1 to WT mice reversed body weight gain, normalized glucose tolerance and intestinal permeability in association with prevention of alteration of the colon mucus layer. We can conclude that the n-3 PUFA-mediated alterations of gut microbiota contribute to the prevention of metabolic syndrome in fat-1 mice and may represent a promising strategy to prevent metabolic disease and preserve a lean phenotype.
\end{abstract}

Keywords: Dietary obesity / n-3 polyunsaturated fatty acids / metabolic endotoxemia / microbiota / fecal transplantation / fat-1 mice

Résumé - Contribution du microbiote intestinal aux effets bénéfiques des acides gras polyinsaturés en n-3 lors de l'obésité alimentaire chez la souris fat-1. L'obésité, aujourd'hui considérée comme une pandémie affectant plus de 650 millions de personnes à travers le monde, est une pathologie complexe principalement associée à un excès d'apport énergétique et des modifications des habitudes alimentaires favorisant l'ingestion d'aliments riches en acides gras saturés et en sucres. Cette pathologie multifactorielle est liée entre autre à une inflammation chronique de bas grade. En effet, un régime obésogène entraîne une dysbiose du microbiote intestinal conduisant à une augmentation de la perméabilité de l'intestin (en partie attribuée à une diminution de l'expression des protéines de jonctions serrées) conduisant à une augmentation du taux circulant de lipopolysaccharides (LPS) définie sous le terme d'endotoxémie métabolique. Différentes études ont montré que les acides gras polyinsaturés (AGPI) en n-3 alimentaires participent à la prévention de l'obésité et de l'insulino-résistance via en particulier la production de médiateurs lipidiques

\footnotetext{
it Contribution to the Topical Issue "Microbiota, Nutrition and Lipids: Consequences on Health".

敌 The French version is available in "Supplementary Material".

*Correspondence: jerome.bellenger@u-bourgogne.fr
} 
résolvants. Alors que des données suggèrent que les AGPI en n-3 peuvent moduler le microbiote intestinal, d'autres études ne montrent aucun effet de traitements aux n-3 sur la dysbiose intestinale. Dans ce travail, nous avons montré que sous régime hypercalorique obésogène, comparativement à des souris de type sauvage, les souris transgéniques fat-1 (aux teneurs tissulaires élevées en AGPI en n-3 conséquence de la synthèse constitutive d'AGPI en n-3 à partir des AGPI en n-6) résistent à l'obésité alimentaire et aux désordres métaboliques associés, conserve une fonction barrière de l'intestin efficace et présente un microbiote de plus grande diversité phylogénique. De plus, une transplantation fécale de souris fat-1 à des souris de type sauvage placées sous régime hyperlipidique obésogène est capable de limiter la prise de poids, de normaliser la tolérance au glucose et la perméabilité intestinale avec une prévention de l'altération de la couche de mucus colique. Nous en concluons qu'une modulation du microbiote intestinal par les AGPI en n3 contribue à la prévention du syndrome métabolique chez la souris fat-1 et pourrait représenter une stratégie prometteuse pour prévenir le développement de maladies métaboliques et préserver un phénotype mince (The full text is available in French on https://www.ocl-journal.org/10.1051/ocl/2021006/olm).

Mots clés : Obésité alimentaire / acides gras polyinsaturés en n-3 / endotoxémie métabolique / microbiote / transplantation fécale

\section{Introduction}

Nowadays, overweight and obesity (a complex pathology associated with numerous metabolic disorders) is a major public health problem in both industrialised and major developing countries. Obesity is a multifactorial disease with 3.4 million related deaths per year and is the 5 th cause of mortality worldwide. Internationally, in $2017,12 \%$ of adults exhibited a BMI (body mass index) greater than $30 \mathrm{~kg} / \mathrm{m}^{2}$ and this prevalence has doubled since the 1980's (Afshin et al., 2017). The French cohort study "Constances", recently concluded that $15.7 \%$ of the adults over 30 are obese (Feral-Pierssens et al., 2018). The 55 to 64 age group is particularly at risk, with $20 \%$ of obese people.

For several years, obesity-related intestinal permeability disorders have been seriously studied. Indeed, intestinal hyperpermeability has been evidenced both in genetically modified obese mouse models or animals fed high-calorie diets (Moreira et al., 2012; Johnson et al., 2015). When studies have shown a large alteration in the function of the intestinal tight junctions (Chelakkot et al., 2018; Thaiss et al., 2018) others have shown the involvement of the intestinal microbiota (Cani et al., 2008). While it is widely recognized today that highcalorie obesogenic diets are responsible for intestinal hyperpermeability in mouse models, human data often remain contradictory. For example, Brignardello et al. observed no change in intestinal permeability in obese people (Brignardello et al., 2010); whereas a similar study reported alterations in intestinal gut barrier function of obese patients (Teixeira et al., 2012). Moreover, the European project MétaCardis, initiated in 2012, has evidenced a lower microbiota diversity in overweight people and this impoverishment worsens with obesity (Vieira-Silva et al., 2020).

Several studies, in both mouse and human, indicate that an n-3-enriched diet appears to improve intestinal gut barrier function and decrease chronic inflammation, in the context of high-fat high-calorie diets. The objective of our in vitro and in vivo studies presented in this work is to characterize the effects of $n-3$ polyunsaturated fatty acids (PUFA) on intestinal permeability, their impact on gut microbiota and the mucus secretion associated with enterocytes protection in a context of dietary obesity.

\section{Obesity and low-grade inflammation: microbiota and lipopolysaccharides involvement}

Among the environmental issues of obesity, intestinal microbiota is a factor that has been largely studied over the last fifteen years. Indeed, throughout the consumption of a highcalorie obsogenic diet, an intestinal microbiota dysbiosis - both qualitative and functional alterations of the microbiota appears with a decrease in its diversity (Turnbaugh et al., 2008; Gérard, 2016). The microbiota, made up of approximately $10^{14}$ bacteria living in the gastrointestinal tract and specific to each individual, is divided in four major phyla of bacteria (Firmicutes, Bacteroidetes, Actinobacteria and Proteobacteria), Firmicutes and Bacteroidetes being the most represented.

Intestinal microbiota also contributes to maintain the integrity of the intestinal barrier function. This latter, is on the one hand covered with tight junction proteins (Zonula Occludens (ZO)-1 and Claudin1 for instance) which mainly contribute to the anchoring of the epithelial cells to the endothelial cells and the microbiota on the other hand, its alteration (dysbiosis), leads to an increase of the intestinal permeability, facilitating the passage of lipopolysaccharides (LPS), membrane components of gram-negative bacteria, from the gut lumen to the bloodstream. This LPS crossing, so-called metabolic endotoxemia, triggers an in situ inflammatory process but also in tissues such as liver, muscle or adipose tissue (Saad et al., 2016). Indeed, LPS bind to TLR (Toll Like Receptor) 4 belonging to the TLR ubiquitous receptor family that function as molecular recognition interfaces for pathogens and lead to a protective immune response (Płóciennikowska et al., 2015). This TLR4 receptor activation, expressed on the surface of many cells such as immune cells, liver, muscle and adipocytes, elicits a metabolic inflammatory response through the production of pro-inflammatory cytokines involved in the inflammation of peripheral tissues related to obesity and associated metabolic disorders. In adipocytes for example, this inflammatory response occurs through the activation of the nuclear factor NF-kB and overexpress pro-inflammatory genes leading to the release of cytokines (Davis et al., 2008). Thus, it has been shown that the targeted TLR4 or its CD-14 (cluster of differentiation 14) gene deletion protects mouse from dietary- 


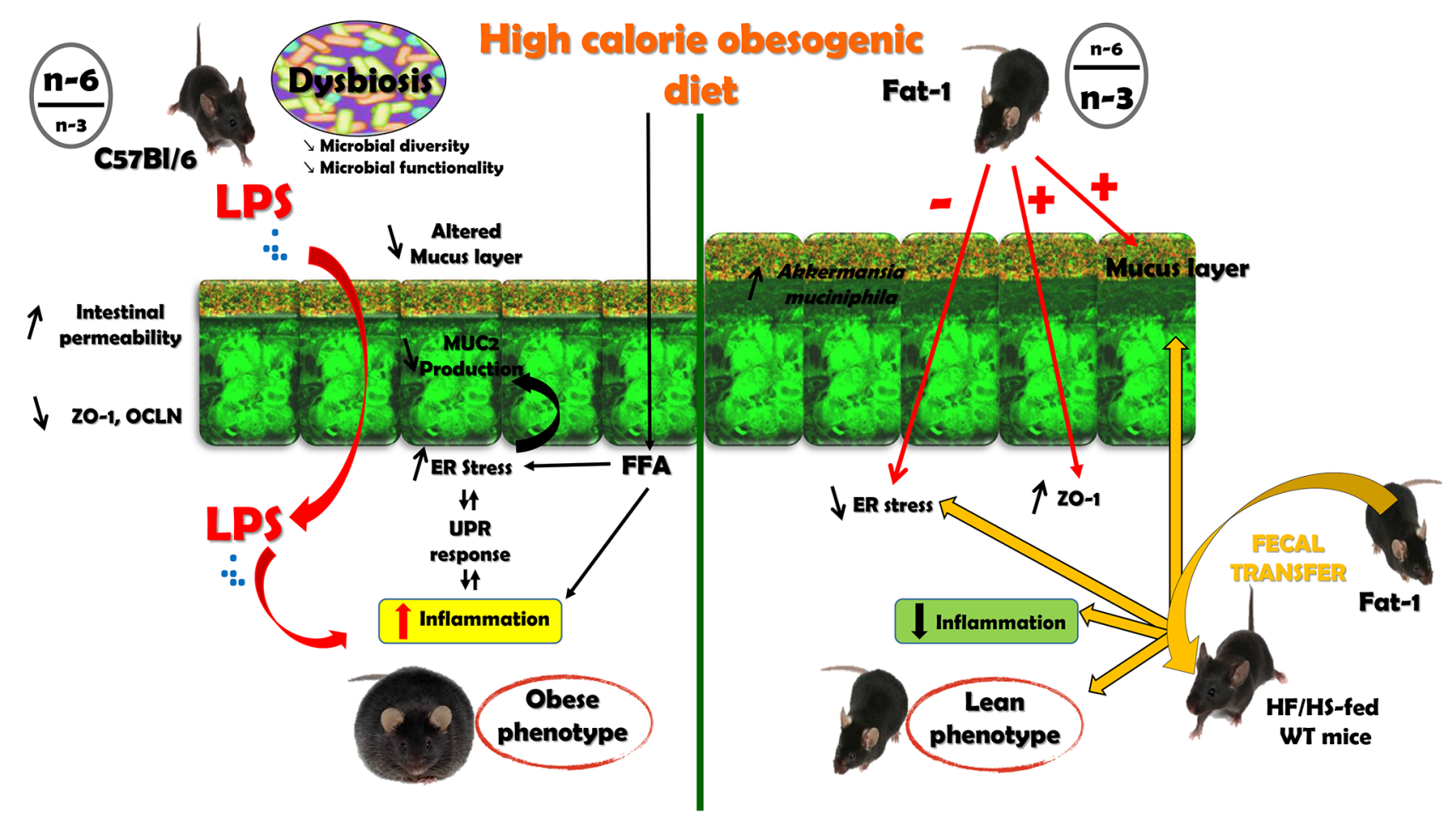

Figure 1. High-fat high-calorie-fed wild-type mice exhibit a gut microbiota dysbiosis and an increased permeability of the intestinal epithelium partly due to a lowered expression of the tight junction proteins but also to a decrease of the integrity of the mucus layer in relation to endoplasmic reticulum stress. This intestinal hyperpermeability facilitates the crossing of LPS leading to low-grade inflammation observed in obese individual. Conversely, we evidenced that fat-1 mice are protected against dysbiosis and intestinal hyperpermeability by a higher expression of tight junction proteins and an unaltered colonic mucus layer, which might be explained at least by the fact that a prevention of the reticulum stress and a higher proportion of Akkermansia muciniphila were observed in fat-1 mice which display a lean phenotype. In addition, the ceacal microbiome transplantation of fat-1 mice to wild-type mice totally prevented high-fat-induced alteration of the colonic mucus layer and endoplasmic reticulum stress in colonic tissues. FFA: free fatty acids; HF/HS: high fat/high sucrose; LPS: lipopolysaccharides; MUC2: mucin 2; OCLN: occludine; ER: endoplasmic reticulum; UPR: unfolded protein response; WT: wild-type; ZO-1: zonula occludens-1.

induced obesity (Davis et al., 2008). Furthermore, it has been observed in mice that the infusion of LPS for 4 weeks mimics the inflammatory metabolic effects induced by consumption of a high-fat and high-calorie diet (Cani et al., 2007).

\section{Impact of n-3 polyunsaturated fatty acids on intestinal permeability and the intestinal microbiota in the context of dietary obesity}

Numerous studies, both in human and animal, have shown that dietary n-3 PUFA are able to participate in the prevention of obesity and insulin resistance notably through the production of pro-resolving lipid mediators (White and Marette, 2014). Nutrigenomic approaches have highlighted cardioprotective effects of n-3 PUFA which can be attributed to a decrease in pro-inflammatory markers, a higher synthesis of pro-resolving lipid mediators and a modulation of genes involved in the synthesis and oxidation of fatty acids (Davidson 2006). Thus, it has recently been shown that dietary supplementation with eicosapentaenoic acid (EPA) increases liver content in resolvin (Rv) E1, RvE2, RvD1 and $\mathrm{RvD} 2$ in high-fat high-calorie-fed mice resulting in a decrease in the hepatic pro-inflammatory status of these animals (Echeverría et al., 2019). Other lipid mediators derived from long chain (LC) n-3 PUFA (as protectins and maresins) are also known to dampen inflammation in a context of dietary obesity. Finally, a recent study evidenced that the administration of protectin D1 (PD1) to $d b / d b$ mice remarkably improve their insulin sensitivity (Li et al., 2014). Nevertheless, several studies also evidenced heterogeneous (or contradictory) effects of n-3 PUFA on obesity. Indeed, some of them support that these fatty acids (FA) can significantly decrease body fat mass whereas others show no significant effect on body weight loss even if the accumulation of fat mass is decreased in animals supplemented in n-3 PUFA. However, a very recent metaanalysis showed that no beneficial effects could be attributed to these FA (Aung et al., 2018).

Dietary n-3 PUFA, through their impact on intestinal integrity, are able to diminish colitis and colon immunodisorders by increasing barrier function of the intestinal epithelium in several animal models. Indeed, EPA has been shown to increase the expression of tight junction proteins as well as the trans-epithelial electrical resistance (TEER), thus decreasing the permeability of intestinal endothelial cells (Jiang et al., 1998). Although very few studies have evaluated the impact of n-3 PUFA on metabolic endotoxemia, the intestinal barrier function seems to be modulated differently depending on the nature of lipids ingested. Mani et al. have evidenced, in swine, that fish oil (rich in n-3 PUFA such as 
EPA and DHA, docosahexaenoic acid) decreases postprandial endotoxemia, that coconut oil (rich in saturated FA) increases endotoxemia whereas olive oil (rich in insaturated FA) has no effect on the endotoxin permeability (Mani et al., 2013).

It has recently been suggested that n-3 PUFA could influence gut microbiota content. A nutritional study by Andersen and coworkers shows, in human, that compared to sunflower oil, a fish oil dietary supplementation impacts on most bacterial phyla (Andersen et al., 2011). In mice, Liu and coworkers observed deep changes in the intestinal microbiota depending of the degree of unsaturation of dietary FA. (Liu et al., 2012). They showed that the proportion of Bactéroidetes was reduced by $28 \%$ in a diet rich in saturated FA, by $10 \%$ in a diet rich in n-3 PUFA and by $12 \%$ with a diet rich in n-6 PUFA. At the family level, the authors also observed a significant decrease of the proportion of Porphyromonadaceae in mice fed with the n-6-enriched diet and of Lachnospiraceae in mice fed with the diet enriched in saturated FA. Moreover, Yu and coworkers have shown that fish oil can lead to deep alteration of the gut microbiota notably by suppressing the growth of Helicobacter and Firmicutes (Yu et al., 2014), known to be strongly involved in the etiology of peptic ulcer and obesity. Finally, a study has evidenced opposite effects of n-6 and n-3 PUFA on metabolic endotoxemia, depending on the hostmicrobiota relationship (Kaliannan et al., 2015). These studies, specifically focusing on these issues, confirm that a link exists between the dietary FA content and their impact on gut microbiota.

Taken together, these results reveal that the modulation of the gut barrier function by n-3 PUFA still remains poorly understood and that the means by which these fatty acids are able to fight against the increase of gut permeability, observed in a context of dietary obesity, still requires further studies. Nevertheless, the complexity of targeting the specific effects of $\mathrm{n}-3$ PUFA is reinforced by the fact that many confounding factors related to the experimental procedures have also been noted in these studies. Consequently, the use of a model avoiding the use of diets appears necessary to prevent these biases and to clarify the interpretations.

The fat- 1 transgenic mouse model has been created by the random insertion into the genome of a C57BL/6 mouse of the fat-1 gene, present in Caenorabditis elegans, encoding a desaturase capable of converting n-6 PUFA to n-3 PUFA. It then represents an alternative approach that allows 1) to replace the use of diets underlying numerous dietary confounding factors and 2) to rapidly and efficiently increase cellular levels of n-3 PUFA while reducing the n-6-to-n-3 ratio (n-6-to-n-3 $=2$ to 5), compared with wild-type mice (n-6-to-n$3=20$ to 50 ). Moreover, in our studies the use of homozygous fat-1 transgenic mice is crucial. Indeed, studies using heterozygous animals (White et al., 2010) have shown that these mice fed an obesogenic high-calorie diet (55\% kcal) developed a dietary obesity comparable to that of wild-type mice while other studies using homozygous mice show a dietary prevention of obesity (Li et al., 2014; Bidu et al., 2018). It can therefore be assumed that the presence of the transgene on a single allele does not appear to be sufficient to prevent the weight gain of these mice. Therefore, endogenous enrichment of fat- 1 mice in n-3 PUFA depends on their conversion rate of n-6 PUFA to n-3 PUFA. It has also been reported that this conversation rate is also strongly depending on the supply in n-6 PUFA substrate (Li et al., 2014).

We then used this mouse model to attempt to answer the following questions: does tissue enrichment in n-3 PUFA protect fat-1 mice from dietary obesity and associated metabolic disorders? Does such a prevention occur, among other things, through a decrease in intestinal permeability and metabolic endotoxemia coupled with a gut microbiota modulation?

\section{The modulation of gut microbiota by endogenous LC n-3 PUFA protects fat-1 mice against dietary obesity and associated metabolic disorders}

To address these questions, wild-type and transgenic fat-1 mice were fed a cafetaria-like diet $(45 \%$ of the energy intake brought by lipids) for twelve weeks. Consistent with previous studies (Kim et al., 2012; Li et al., 2014), we showed that fat-1 mice were protected against weight gain induced by an obesogenic high-calorie diet, partly due to an increase in energy expenditure supported by an overexpression of the uncoupling protein 1 (UCP1) in adipose tissue (Bidu et al., 2018). We also observed that fat-1 mice were also protected against metabolic disorders associated with obesity, such as hepatic steatosis, whole-body glucose intolerance and adipose tissue inflammation. Moreover, compared to wild-type mice, transgenic fat-1 mice exhibit, in association with a huge decrease in the n-6-to-n3 ratio, an increase of the skeletal muscle glucose uptake occurring independently of the presence of insulin. This protective effect could be attributed to increased docosanoid biosynthesis in muscle of fat-1 fed a high-calorie diet. Indeed, White et al. evidenced that the proresolving lipid mediator, resolvin PD1 was increased by $176 \%$ in the tissue of fat- 1 mice versus wild-type mice (White and Marette, 2014). Our work also evidenced that, when fed a high-fat high-calorie diet, fat-1 mice are protected against intestinal permeability and associated endotoxemia (Fig. 1). Thus, tissue enrichment in n-3 LCPUFA prevents decreased expression of tight junction protein, such as $\mathrm{ZO}-1$, and maintains the integrity of the intestinal gut barrier (Bidu et al., 2018).

Microbiota is known to be a major player in intestinal and energy homeostasis (Backhed et al., 2004), we then studied its involvement in the preventive effects of n-3 LCPUFA in fat-1 mice during obesity and associated alteration. Besides significant differences in cecal microbiota between the two genotypes, fat-1 mice display significantly greater bacterial diversity (particular abundance of the Bacteroides genus) than wild-type mice when both were fed the obesogenic highcalorie diet. We also observed an increase in the abundance of Akkermansia muciniphila in fat-1 mice (Bidu et al., 2018), reported to be protective against obesity and associated metabolic (Everard et al., 2013) and intestinal disorders (Plovier et al., 2017). Modulation of the microbiota by a n-3 tissue enrichment could partly be explained by the fact that intestinal bacteria would enriched with n-3 PUFA by "feeding" continuously renewed epithelial cells, which would consequently change the composition and functions of the microbiota. In addition, n-3-tissue enrichment could also contribute 
to the change of microbiota community by modulating the production of antimicrobial peptides such as defensin, lysozymes or colon mucus secretion.

In order to confirm a cause-and-effect relationship between changes in cecal microbiota and the observed metabolic trait, we transferred fat-1 fecal microbiota to wild-type mice previously treated with a single dose of streptomycin to highly reduce the resident microbiota and facilitate the implantation of the transplanted microbiota. We have shown that the transfer of fat-1 microbiota protects wild-type mice against weight gain and metabolic and intestinal alterations elicited by 18 weeks of highfat high-calorie diet (Bidu et al., 2018). These microbiota transfer experiments led us to demonstrate the preventive effects of intestinal microbiota modulated by n-3 LCPUFA in dietinduced obesity and associated metabolic disorders (Fig. 1).

\section{N-3 PUFA and colonic mucus}

Besides the epithelial component, we then focused on the intestinal mucus layer, another important component of the intestinal barrier function at the interface between intestinal microbiota and colonic endothelial cells. It is now wellestablished that under an obesogenic diet rich in saturated FA (specially palmitic acid), the intestinal mucus layer, whose the glycoprotein MUC2 (mucin-2) secreted by goblet cells is the main protein, is drastically altered, which then enable higher intestinal permeability and metabolic endotoxemia establishment (Cani et al., 2007; Gulhane et al., 2016).

In addition, it has been shown that high-fat and highcalorie diet causes endoplasmic reticulum (ER) stress in intestinal epithelial cells, lowering MUC2 production and consequently alter the integrity of the mucus layer, which increase the leakage of LPS leading to low-grade inflammation. Free FA provided by the diet (but also those released from adipose tissue throughout lipolysis) seem to be direct ER stress activators in colonic goblet cells secreting mucus (Gulhane et al., 2016). To our knowledge, no study has examined the potential preventive effects of n-3 LCPUFA regarding the alteration of the mucus layer under obesogenic conditions. For that, two approaches have been developed: first, in vitro using human colonic epithelial cells (LS174T), and second in vivo (unpublished data) using the fat-1 transgenic mouse model.

In the first approach, we showed that treating LS174T cells with palmitic acid (PAL) decreases MUC2 secretion in association with an increase of ER stress (as produced by pharmacological inducers as thapsigargin or tunicamycin (Guha et al., 2017), whereas EPA and DHA are able to prevent ER stress and to restore MUC2 secretion (Escoula et al., 2019). We then showed that ER stress inhibition by 4-PBA (an ER stress inhibitor) maintained MUC2 secretion despite the presence of PAL, thus confirming that the altered MUC2 release observed in PAL-treated LS174T cells occurs mainly through the triggering of ER stress (Escoula et al., 2019). The well-known anti-inflammatory effects of EPA and DHA may also be involved in the modulation of altered goblet cell homeostasis and decreased mucin production. Indeed, resolvins - derived from EPA and $\mathrm{DHA}$ - due to their antiinflammatory properties, have also been suggested to attenuate ER stress-induced apoptosis in HepG2 cells, mainly through the JNK pathway (Jung et al., 2014). Moreover, it has also been suggested that n-3 PUFA suppression of ER stress was partly due to AMP-activated protein kinase (AMPK) activation. To support this hypothesis, compound C (an AMPK inhibitor) is able to block the effects of DHA in PAL-induced ER stress (Yang et al., 2017).

By contrast with studies previously reported in the literature (Katsoulieris et al., 2009; Zhang et al., 2011), we have not observed any preventive effect of the $\alpha$-linolenic acid (ALA) regarding ER stress, nor the altered MUC2 secretion induced by PAL. Several studies reported distinct effects of DHA and ALA, particularly on oxidation induced by PAL in other cell types, such as C2C12 myoblasts (Pinel et al., 2016) and L6 myotubes (Pimenta et al., 2008), where partial and total oxidation were decreased during PAL treatment, which was restored by EPA and DHA, but not by ALA. Moreover, besides alterations in this oxidation, PAL treatment has also been shown to increase the formation of lipotoxic compounds, such as diglycerides and ceramides (Chavez et al., 2003) which can be lowered by EPA and DHA. These last also enhance triglyceride synthesis, and preferentially address PAL to mitochondrial oxidation. In agreement with Pinel and coworkers (Pinel et al., 2016), ALA failed to prevent PAL incorporation into cytotoxic diglycerides, and then to reduce the related ER stress activation and finally MUC2 production. These results indicate that, unlike EPA and DHA, ALA does not, by itself, have a direct effect in preserving MUC2 secretion and mucus layer thickness. Nevertheless, the relevance of our in vitro data remains to be explored in vivo in a context of dietary obesity, as well as the efficiency ofn-3PUFA in alleviating the decrease of the thickness of the intestinal mucus layer and consequently preserving gut barrier integrity.

Consequently, in a second approach (not yet published) we first confirmed what was described in the literature: obese wild-type mice, when fed a high fat-high-calorie diet, exhibit an alteration of the mucus layer. Furthermore, we showed for the first time a preserved integrity of the mucus layer in highfat high-calorie-fed fat-1 mice. This was accompanied by the maintainance of MUC2 and Klf4 (a goblet cell differentiation factor) expressions and an alleviation of the colonic reticulum stress. In order to understand the potential role of the intestinal microbiota of fat- 1 mice in terms of preventing alterations of the colonic mucus layer, we evaluated the impact of transferring fat-1 microbiome to recipient wild-type mice fed an high-calorie obesogenic diet on the preserved integrity of the mucus layer. We evidenced (data not yet published) that this transfer of microbiota was not only able to alleviate weight gain, improve glucose tolerance and gut barrier function, but also to remarkably preserve the integrity of the inner colonic mucus layer in mice fed the high-fat obesogenic diet. Conversely, transfer of wild-type microbiote has no preventive effect in wild-type mice fed the high-calorie obesogenic diet. We have also shown that this preservation of the colonic mucus layer after transferring fat-1 microbiota is related to a maintained expression of MUC2 and Klf4 and a prevention of the colonic ER stress (Fig. 1).

\section{Conclusion}

Taken together, these data allowed us to confirm the important role of n-3 LCPUFA in addressing the issue of 
obesity and to mainly highlighting the preventive role of the intestinal microbiota of fat- 1 mice. We were also able to understand how the intestinal microbiota of fat- 1 transgenic mice, whose tissues are endogeneously enriched in LCPUFA, participates in the prevention of alterations of the gut barrier and, ultimately, of dietary obesity. In the present work, we have evidenced the ability of the microbiota of fat-1 mice, modulated by the n-3 FA, to maintain the integrity of the colonic mucus layer under high-calorie obesogenic conditions (Fig. 1), thus opening new opportunities for the use of fecal transplantations for therapeutic applications in the management of metabolic diseases.

Furthermore, these results open up new research avenues, such as the identification of bacterial species or bacterial metabolites, involved in the lean phenotype transmission of fat-1 mice and their resistance to metabolic diseases.

\section{Supplementary Material}

French version.

The Supplementary Material is available at https://www.ocljournal.org/10.1051/ocl/2021006/olm.

Acknowledgment. This work has been supported by INSERM (National Institute of Health and Medical Research), the Regional Council of Burgundy, the European Regional Development Fund, the University of Burgundy, the Fondation de France, and by the National Research Agency under the program "Investissements d'Avenir" with the reference ANR11-LABX-0021-01-LipSTIC LabEx. This project was also supported by Valorex (Combourtillé, France) and the National Association for Technological Research (ANRT) with CIFRE thesis funding.

Conflicts of interest. The authors declare having no conflict of interest.

\section{References}

Afshin A, Forouzanfar MH, Reitsma MB, et al. 2017. Health Effects of Overweight and Obesity in 195 Countries over 25 Years. N Engl J Med 377(1): 13-27.

Andersen AD, Molbak L, Michaelsen KF, Lauritzen L. 2011. Molecular fingerprints of the human fecal microbiota from 9 to 18 months old and the effect of fish oil supplementation. J Pediatr Gastroenterol Nutr 53(3): 303-309.

Aung T, Halsey J, Kromhout D, et al. 2018. Associations of Omega-3 Fatty Acid Supplement Use With Cardiovascular Disease Risks: Meta-analysis of 10 Trials Involving 77917 Individuals. JAMA Cardiol 3(3): 225-234.

Backhed F, Ding H, Wang T, et al. 2004. The gut microbiota as an environmental factor that regulates fat storage. Proc Natl Acad Sci USA 101(44): 15718-15723.

Bidu C, Escoula Q, Bellenger S, et al. 2018. The Transplantation of omega3 PUFA-Altered Gut Microbiota of fat-1 Mice to WildType Littermates Prevents Obesity and Associated Metabolic Disorders. Diabetes 67(8): 1512-1523.

Brignardello J, Morales P, Diaz E, Romero J, Brunser O, Gotteland M. 2010. Pilot study: alterations of intestinal microbiota in obese humans are not associated with colonic inflammation or disturbances of barrier function. Aliment Pharmacol Ther 32 (11-12): 1307-1314.

Cani PD, Amar J, Iglesias MA, et al. 2007. Metabolic endotoxemia initiates obesity and insulin resistance. Diabetes 56(7): 17611772.

Cani PD, Bibiloni R, Knauf C, et al. 2008. Changes in gut microbiota control metabolic endotoxemia-induced inflammation in high-fat diet-induced obesity and diabetes in mice. Diabetes 57(6): 1470 1481.

Chavez JA, Knotts TA, Wang LP, et al. 2003. A role for ceramide, but not diacylglycerol, in the antagonism of insulin signal transduction by saturated fatty acids. J Biol Chem 278(12): 10297-10303.

Chelakkot C, Ghim J, Ryu SH. 2018. Mechanisms regulating intestinal barrier integrity and its pathological implications. Exp Mol Med 50(8): 103.

Davidson MH. 2006. Mechanisms for the hypotriglyceridemic effect of marine omega-3 fatty acids. Am J Cardiol 98(4a): 27i-33i.

Davis JE, Gabler NK, Walker-Daniels J, Spurlock ME. 2008. Tlr-4 deficiency selectively protects against obesity induced by diets high in saturated fat. Obesity (Silver Spring) 16(6): 1248-1255.

Echeverría F, Valenzuela R, Espinosa A, et al. 2019. Reduction of high-fat diet-induced liver proinflammatory state by eicosapentaenoic acid plus hydroxytyrosol supplementation: involvement of resolvins RvE1/2 and RvD1/2. J Nutr Biochem 63: 35-43.

Escoula Q, Bellenger S, Narce M, Bellenger J. 2019. Docosahexaenoic and Eicosapentaenoic Acids Prevent Altered-Muc2 Secretion Induced by Palmitic Acid by Alleviating Endoplasmic Reticulum Stress in LS174T Goblet Cells. Nutrients 11(9): 2179.

Everard A, Belzer C, Geurts L, et al. 2013. Cross-talk between Akkermansia muciniphila and intestinal epithelium controls dietinduced obesity. Proc Natl Acad Sci USA 110(22): 9066-9071.

Feral-Pierssens AL, Carette C, Rives-Lange C, et al. 2018. Obesity and emergency care in the French CONSTANCES cohort. PLoS One 13(3): e0194831.

Gérard P. 2016. Gut microbiota and obesity. Cell Mol Life Sci 73(1): $147-162$.

Guha P, Kaptan E, Gade P, Kalvakolanu DV, Ahmed H. 2017. Tunicamycin induced endoplasmic reticulum stress promotes apoptosis of prostate cancer cells by activating mTORC1. Oncotarget 8: 68191-68207.

Gulhane M, Murray L, Lourie R, et al. 2016. High Fat Diets Induce Colonic Epithelial Cell Stress and Inflammation that is Reversed by IL-22. Sci Rep 6: 28990.

Jiang WG, Bryce RP, Horrobin DF, Mansel RE. 1998. Regulation of tight junction permeability and occludin expression by polyunsaturated fatty acids. Biochem Biophys Res Commun 244(2): 414-420.

Johnson AM, Costanzo A, Gareau MG, et al. 2015. High fat diet causes depletion of intestinal eosinophils associated with intestinal permeability. PLoS One 10(4): e0122195.

Jung TW, Hwang HJ, Hong HC, et al. 2014. Resolvin D1 reduces ER stress-induced apoptosis and triglyceride accumulation through JNK pathway in HepG2 cells. Mol Cell Endocrinol 391(1-2): 30-40.

Kaliannan K, Wang B, Li XY, Kim KJ, Kang JX. 2015. A hostmicrobiome interaction mediates the opposing effects of omega-6 and omega-3 fatty acids on metabolic endotoxemia. Sci Rep 5: 11276.

Katsoulieris E, Mabley JG, Samai M, Green IC, Chatterjee PK. 2009. Alpha-Linolenic acid protects renal cells against palmitic acid lipotoxicity via inhibition of endoplasmic reticulum stress. Eur $J$ Pharmacol 623(1-3): 107-112. 
Kim EH, Bae JS, Hahm KB, Cha JY. 2012. Endogenously synthesized n-3 polyunsaturated fatty acids in fat- 1 mice ameliorate high-fat diet-induced non-alcoholic fatty liver disease. Biochem Pharmacol 84(10): 1359-1365.

Li J, Li FR, Wei D, et al. 2014. Endogenous omega-3 polyunsaturated fatty acid production confers resistance to obesity, dyslipidemia, and diabetes in mice. Mol Endocrinol 28(8): 1316-1328.

Liu T, Hougen H, Vollmer AC, Hiebert SM. 2012. Gut bacteria profiles of Mus musculus at the phylum and family levels are influenced by saturation of dietary fatty acids. Anaerobe 18(3): 331-337.

Mani V, Hollis JH, Gabler NK. 2013. Dietary oil composition differentially modulates intestinal endotoxin transport and postprandial endotoxemia. Nutr Metab (Lond) 10(1): 6.

Moreira AP, Texeira TF, Ferreira AB, Mdo Peluzio C, Alfenas Rde C. 2012. Influence of a high-fat diet on gut microbiota, intestinal permeability and metabolic endotoxaemia. BrJ Nutr 108(5): 801809.

Pimenta AS, Gaidhu MP, Habib S, et al. 2008. Prolonged exposure to palmitate impairs fatty acid oxidation despite activation of AMPactivated protein kinase in skeletal muscle cells. $J$ Cell Physiol 217(2): 478-485.

Pinel A, Rigaudiere JP, Laillet B, et al. 2016. N-3PUFA differentially modulate palmitate-induced lipotoxicity through alterations of its metabolism in C2C12 muscle cells. Biochim Biophys Acta 1861 (1): 12-20.

Płóciennikowska A, Hromada-Judycka A, Borzęcka K, Kwiatkowska K. 2015. Co-operation of TLR4 and raft proteins in LPSinduced pro-inflammatory signaling. Cell Mol Life Sci 72(3): $557-581$.

Plovier H, Everard A, Druart C, et al. 2017. A purified membrane protein from Akkermansia muciniphila or the pasteurized bacterium improves metabolism in obese and diabetic mice. Nat Med 23(1): 107-113.
Saad MJ, Santos A, Prada PO. 2016. Linking Gut Microbiota and Inflammation to Obesity and Insulin Resistance. Physiology (Bethesda) 31(4): 283-293.

Teixeira TF, Souza NC, Chiarello PG, et al. 2012. Intestinal permeability parameters in obese patients are correlated with metabolic syndrome risk factors. Clin Nutr 31(5): 735-740.

Thaiss CA, Levy M, Grosheva I, et al. 2018. Hyperglycemia drives intestinal barrier dysfunction and risk for enteric infection. Science 359(6382): 1376-1383.

Turnbaugh PJ, Backhed F, Fulton L, Gordon JI. 2008. Diet-induced obesity is linked to marked but reversible alterations in the mouse distal gut microbiome. Cell Host Microbe 3(4): 213-223.

Vieira-Silva S, Falony G, Belda E, et al. 2020. Statin therapy is associated with lower prevalence of gut microbiota dysbiosis. Nature 581(7808): 310-315.

White PJ, Marette A. 2014. Potential role of omega-3-derived resolution mediators in metabolic inflammation. Immunol Cell Biol 92(4): 324-330.

White PJ, Arita M, Taguchi R, Kang JX, Marette A. 2010. Transgenic restoration of long-chain n-3 fatty acids in insulin target tissues improves resolution capacity and alleviates obesity-linked inflammation and insulin resistance in high-fat-fed mice. Diabetes 59: 3066-3073.

Yang W, Chen X, Chen M, et al. 2017. Fish oil supplementation inhibits endoplasmic reticulum stress and improves insulin resistance: involvement of AMP-activated protein kinase. Food Funct 8(4): 1481-1493.

Yu HN, Zhu J, Pan WS, et al. 2014. Effects of fish oil with a high content of n-3 polyunsaturated fatty acids on mouse gut microbiota. Arch Med Res 45(3): 195-202.

Zhang Y, Dong L, Yang X, Shi H, Zhang L. 2011. alpha-Linolenic acid prevents endoplasmic reticulum stress-mediated apoptosis of stearic acid lipotoxicity on primary rat hepatocytes. Lipids Health Dis 10: 81 .

Cite this article as: Bellenger J, Bellenger S, Bourragat A, Escoula Q, Weill P, Narce M. 2021. Intestinal microbiota mediates the beneficial effects of n-3 polyunsaturated fatty acids during dietary obesity. OCL 28: 21 . 